\title{
XI.
}

\section{Le nuove dottrine anti-vitali dopo la guerra}

di

\section{Allessandro Chiappelli,}

\author{
Senatore del Regno.
}

Se è vero che le forme del pensiero speculativo espressero sempre la somma del modo di sentire e di vivere proprio di ogni tempo, non è meraviglia che, nella cultura europea del periodo precedente alla più vasta delle guerre storiche teste terminata, l'idea predominante e maestra a tutte le altre relative ai fatti e sentimenti umani, fosse la volontà sfrenata della vita e della potenza dominatrice; idea questa, variamente espressa ed atteggiata in forme sistematiche nel pensiero inglese, tedesco, francese e italiano degli ultimi tempi; dal Nietzsche allo Spencer, dal Guyau al Bergson ai neonietzschiani e neo-dannunziani d'Italia: da tutti insomma i fautori della forza e gli esaltatori dell'intensità della vita. Comunque s'intendesse teoricamente e scientificamente dai biologi la natura della vita, o come processo di adattamento organico alle condizioni esteriori (H. Spencer), ovvero come principio originalè, spontaneo e creativo (neo-vitalismo del Driesch, del Bunge, del Lodge e degli altri), certo è che la volontà della vita, di per sè stessa, indipendentemente dal contenuto suo onde ha valore, era posta sugli altari, quale forma più eccelsa della moralità, ed atto ed abito solo veramente degno dell'uomo. Mentro i filosofi elevavano l'impulso vitale a dignità di centro vero della realtà universa (biologismo metafisico, Bergsonismo), da altri si predicava e proclamava supremo ideale dell'attività etico-sociale dell'uomo l'affermazione vigorosa e l'espansione della vita in tutte

1) Cfr.; fra gli altri, il libro della Paget, Gospels of Anarchy, London 1908, e fini saggi raccolti da J. Humeker nel volume Egoists, a Book of Supermen, New York 1909. 
le sue forme. Gran parte della letteratura degli ultimi anni fino a Maurice Barrès, che lo stesso Anatole France chiamo ,perfido idealista", al Kipling e al d'Annunzio, aveva celebrata in tutte le forme e cantato in tutti i toni il trionfo della vita e della forza (lo Strenu ou s Lif e) nella varia affigurazione artistica del cosi detto uomo superiore, dell'individuo privilegiato e dominatore ${ }^{1}$ ). Il tipo dell'eroe era identificato con quello del dominatore. E se la guerra ha dimostrato fortunatamente che per la coscienza civile ancora l'eroe è quello chè si sacrifica per gli altri, la persuasione prevalente prima della guerra e fu impulso alla guerra, era che solo e degno di tal nome sia quegli che sacrifica gli altri a sè, cioè il soverchiatore ed oppressore: e il tipo del violento e del potente, del Cesare o del Napoleone, era offerto all'ammirazione e quasi al culto púbblico e magnificato sulle scene, nei romanzi, nelle laudi liriche degli eroi, nelle esposizioni artistiche. Nè era valso che alcuni spiriti liberi e generosi (e non soltanto per affinità e tendenze socialistiche 0 cristiane) avessero protestato contro questa deformazione umana che esaltava, sotto nuove specie, la figura barbarica dell'oppressore; o che altri, come Bernardo Shaw, il quale nella Commedia Major. Barbara aveva pure impersonato cotesto tipo nella figura di Undershaft, nel suo possente dramma Man and Superman avessero additato il vero senso della vita e la pura scaturigine della gioia del vivere veramente umano nell'essere e nel sentirsi l'uomo cooperatore colle forze cosmiche e partecipe del destino e del mistero della natura universa. L'evangelio della forza era oramai la formula prevalente nell'arte come espressione più alta e più degna della vita: e la predicazione etico-sociale chè dell'uomo superiore si estendeva logicamente al popolo superiore e prepossente (Übermensch, Übervolk) preparava quello che altra volta io dissi ${ }^{2}$ ), il diffuso spirito di guerra in tutta la cultura recente. La Germania non fece che precipitare, coll'atto decisivo; questa saturazione, che Dante chiamò stupendamente ,il cielo intento";

si che'l pregno aere in acqua si converse, e l'aragano di guerra proruppe formidabile ma inevitabile.

Ora è ben naturale che dopo tanta devastazione civile e nello squallore dell'ora tragica riappaiano già sull'orizzonte della nuova.

$\left.{ }^{2}\right)$ V. il mio scritto Lo spirito della Guerra, nella Nuova Antologia; 16 giugno 1917: 
cultura lineamenti di dottrine che non solo rinnegano, in nome della giustizia e della civiltà, quella formula della vita ond'era glorificato l'ideale dell'uomo e del popolo dominatore e soverchiatore, ma giungano a volere estirpare la creduta radice essenziale di quella mala pianta, il sentimento stesso e il valore della vita. La guerra mondiale, che può essere stata liberatrice per alcune nazioni come l'Italia, considerata nella sua universalità non è stata che una formidabile conseguenza di quello esaltamento e potenziamento della volontà vitale a volontà di dominio e di prepotenza onde il superuomo eliminava I'uomo, e alla volontà di un nobilissimo popolo credutosi l'eletto rischiava d'immolare il senso e il rispetto della comune umanità: Allontanato, almeno per ora, questo pericolo sovrastante, era legittimo attendersi che alle precedenti concezioni biocentriche, anche nell'ordine del pensiero, succedessero i primi tentativi di una dottrina radicalmente opposta, di una filosofia antivitale o negatrice della volontà stessa della vita, quasi ella sia alcunchè d'irrazionale: ritornando cosi, in forme nuove, all'antico motivo orientale della estinzione della vita e della rinuncia, che dall'antico Buddha scende giù fino all'odierno Rabindranath Tagore ${ }^{3}$ ) o rinnovando la negazione della volontà vitale dello Schopenhauer, col vagheggiare il finale annichilamento di questo malaugurato istinto, per virtù della ragione adulta e della coscienza illuminata oramai dalle dolorose esperienze della storia.

Codesta ripresa di antichi motivi, sintomo di questo stato d'animo di sfiducia e di depressione nelle classi colte dell'Europa occidentale, più diffuso di quanto non si creda, e dilagante dopo gli effetti di tanta guerra, si manifesta, da un láto, nel risorgere di uno scetticismo teorizzato in opere come quella del Renzi, del'Università di Genova ${ }^{4}$ ); e dall'altro nel ridestarsi del problema religioso e morale, del quessito sul valore e la finalità, nella recente letteratura, e segnatamente nel romanzo inglese e nel romanzo russo. Nell'ultimo suo romanzo (The Undying Fire London 1919), il Wells, rinnovando l'antico dibattito del biblico Job, conclude bensì che l'uomo può e deve collaborare con Dio alla creazione d'un mondo migliore e al trionfo del bene, dacchè l'esperienza tragica della guerra non possa andare in tutto

3) Tagore, Nationalism, London 1918, specialmente quello che si dice sul nazionalismo occidentale, p. 3-46; e Personality, London 1917.

4) G. Renzi, Lineamenti di una Filosofia Scettica, Bologna 1919. 
serduta, e lascia ancora aperto l'adito allo spiraglio di un vago ttimismo sociale. Ma il romanziere russo (di cui è stata annunziata a fine) l'Andrejeff, che ha veduta e sentita la morte, prima nella miseria e nell'abbrutimento del servaggio russo sotto lo Zarismo (cfr. il dramma La vita dell'uomo), poi nelle stragi e negli orrori della guerra mondiale, infine nelle violenze del Bolschevismo (Sotto il gioco della Guerra) esprime, in un momento in cui si appresta al suicidio nella Neva, il sentimento della certezza dell'indistruttibilità umana, della immortalità della sua vita personale, della continuità della propria esistenza nella morte: la quale, in uno degli ultimi suoi romanzi, il Lazzaro risuscitato ${ }^{5}$ ), vien rappresentato tragicamente come il vacuo, il non ente, il silenzio, riproducendo lo stesso motivo di Hamlet; the rest is silence.

Ma più chiaro segno di questa profonda crisi morale è il delinearsi di tentativi ancora iniziali ed ingenui nelle loro movenze e profetici nelle loro tendenze, ma non scevri di accenti originali ${ }^{6}$ ), insistono, da un lato sopra una visione organica del processo storico; e dall'altro e principalmente, sopra la persuasione di una antitesi insuperabile fra la vita, intesa come istinto di usurpazione e di sopraffazione, e la ragione (il Logos umano); facoltà universale, che dovrà éssere vittrice da ultimo di codesto istinto fatale ed illusorio. Non è dubbio, difatti, che, postosi ora in qualsiasi modo fine alla guerra e pei resultati finora maturati nelle trattative di pace, il momento storico presente sembri segnare l'atto di cessione del primato civile che dall'Europa passa all'America, e lo spostamento quasi dell'asse délla civiltà 0 del centro storico dall'antico al nuovo continente. Di questo fatto più che l'esausta Europa pare essere oggi consapevole essa l'America; la quale, per-bocca del Presidente Wilson nell'ultimo messaggio al Congresso nord-americano, proclamava senza reticenze: „l'equilibrio

5) Trad. it. Firenze Valsecchi 1919.

6) Alludo principalmente alle brevi ma notevoli pagine d'uno scrittore il cai „nome ancor molto non suona“, Silvio Pagani, pubblicate nel Coenobium di Lugano (XII, 1919) col titolo Introduzione alla Filosofia dell'Antivita, che praannunziano un'opera sul mondo come volontà antivitale. Ma vedasi già anche A. Lalande, La Dissolution opposée à l'Évolution dans les sciences physiques et morales, Paris, Alcan, 1889; Henri Marconi, Histoire de l'Eेvolution naturelle, Paris-Lugano 1915; Simmel, Philosophische Kultur; 2. Aufl., 1919. 
del mondo è nelle nostre mani, e nelle nostre soltanto . . . . l'America ha salvato l'Europa intervenendo nella guerra, e deve salvarla di nuovo mediante la sua azione nella pace". $\mathrm{Ma}$ in ogni modo l'apparire della cultura transoceanica, finora quasi in tutto riflesso dell'europea ${ }^{7}$ ), sulla scena storica dell'antico continente segna un nuovo passo di quel cammino visibile della storia che procede non diversamente dal corso apparente del sole, cioè dall'Oriente all'Occidente. Certo, non si può parlare altro che in termini vaghi e con indeterminatezza d'analogia, di una infanzia, di una giovinezza, di una maturità, e di un decadimento o vecchiezza dell'umanità civile; e può parer soverchio l'asseverare che la ventura epoca americana segnerà l'età virile della storia rispetto al periodo europeo che ne avrebbe compresa la: lunga giovinezza. Anche nella storia della civiltà europae si alternarono tempi di giovinezza e periodi di senilità: e la civiltà americana si presenta piuttosto oggi con segni di vigor nuovo e d'impeto giovanile, disdegnosa come è del passato e delle tradizioni venerande della storia (onde l'America pare disconoscere i diritti e lá dignità dell'Italia madre), che non con propositi meditati e ponderati, propri di chi sa trarre dall'esperienza storica precedente ammaestramenti a mature e prudenti riforme degli ordini passati. Tanto meno poi io penso si possa assegnare a queste varie epoche storiche una anche approssimativa determinazione cronologica, come pur si è tentato dagli antichi Brahmani fino ai nostri giorni. Ed è lecito altresi dubitare se, dopo la parabola dei secoli, nella sua fase senile l'umanità, stanca ed esausta dal suo lungo e faticoso cammino, debba ritornare a quel lontano Oriente donde mosse, e nelle isole di quel Giappòne, che ora mostra di voler essere parte attiva della nuova storia, o sulle terre della Cina, ridesta dal sonno di secoli, sia per trovare il riposo finale dopo così lunga, intensa, e consumatrice opere di vita. Man non pare si possa revocare almeno in dubbio che il volo dell'umanità abbia pur quella direzione transoceanica, e che la trasmissione pana tenaica della lampäda vitale fra le nazioni civili (per ripetere la vecchia e viva imagine che Lucrezio derivò da Platone) avvenga dell'Asia all'Europa $\theta$ da questa all'America; la quale mentre sta raccogliendo l'eredità altrui, civiltà

7) Mi sia lecito riferirmi al mio scritto: Il nuovo pensiero americano, nella Nuova Antologia, 1916, a proposito del libro del Woodbridge Riley, The american Thought. 
giovine com'è, si prepara ad ardere violenta come in un impetuoso incendio spirituale, a creare torse i più grandi miracoli tecnici di cui sia stato capace il genio dell'uomo, e con superbi progressi industriali, ad elevare la condizione materiale della vita civile ad altezze finora insospettate.

Cercare la ragione naturale di questo movimento civile verso l'occidente in una misteriosa Anticineși ,rotativa della terra che solleciti gli esseri umani, fra gli altri organismi, a spostarsi, individualmente e collettivamente, dall'Oriente all'occaso in senso contrario alla rotazione terrestre ${ }^{\text {(8) }}$; mi sembra un volere illustrare „l'oscuro col più oscuro"; e piuttosto sarebbe a cercarne, non dico la ragione ma la condizione, naturale e storica insieme, in quell'istinto dei popoli che li sospinge a seguire le ,umide vie" dei fiumi e dei mari come veicolo di prosperità civile ${ }^{9}$ ). L'avanzare della civiltà, di fatto, è congiunto strettamente coll'avanzare continuo sulle vie fluviali e marittime, in anelli sempre più larghi $\mathrm{e}$ in ispazi sempre più vasti; dai grandi imperi orientali che fiorirono sulle rive dei grandi fiumi. l'Indo e il Gange, il Tigri, l'Eufrate, il Nilo, alla civiltà occidentale, che prima è mediterranea e più tardi è oceanica. „La civiltà nostra (scrissi altrove) nacque, come in suo nido, nel Mediterraneo orientale, più angusto e disseminato d'isole e di arcipelaghi. Quivi per sette foci il Nilo portava il frutto dell'antichissima civiltà egizia. Qui d'isola in isola, pel tramite di facile navigazione, passarono i fiorenti commerci fenici, i prodotti delle arti, le forme religiose, la lingua armoniosa dell'Ellade: e dall'alto dell'acropoli la divina Atene vedeva fervere la vita del suo popolo nei mari suoi, cosparsi e quasi costellati d'isole innumerevoli. Qui, nel Mediterraneo, dalle montagne dell'estrema Galilea che si affacciano e si protendono sul mare, scese la parola nuova di vita spirituale al mondo; e prima la portò, navigando, la fiera anima di Saulo di Tarso, accendendo via via su tutte le coste mediterranee come tanti lumi ardenti dalla fiamma della sua indomita fede." Ma per l'angusto varco gaditano la civiltà mediterranea si

8) Cosi il Dubois, Les origines natura!les de la Guerre, Lyon 1916.

9) Vedi il mio scritto, già nella Rivista d'Italia; 1901, riprodotto nel volume Voci del nostro tempo. Palermo, Sandron; 1903, p. 119-152; e i classici lavor ivi citati, del Ratzel, Das Meer als Quelle der Völkergröße, München 1900, e del cap. Mahan, The Influence of the Seapower upon the History, London 1900 . 
apri la via verso l'immensurabile oceano; e l'Atlantico parve veramente il gran fiume Oceano dei poemi omerici, sulle cui rive si assisero, scambiandosi i loro prodotti materiali e spirituali, le due grandi parti dell'umanità civile, l'Europa e l'America, quasi due immensi semicori ché levassero concordi il cantico della nuova umanità. Se non chè la. vita civile anela a spazi sempre più vasti, si apre vie sempre nuove: e col taglio dell'istmo di Panama è segnato il nuovo passo ulteriore onde l'America sembra voler condurre la civiltà umana sopra una più larga scena storica, cioè in quell'amplissimo bacino del Pacifico, che sarà forse il teatro della futura opera civile dell'umanità.,

Piuttosto, dunque, che ad un progressivo inaridimento delle fonti della vita, queste linee onde par segnata la via della storia sembrano invece accennare ad una intensificazione crescente di esse, per quanto lontano possan portare le umane previsioni. Se non che quelli che io potrei chiamare i ,nuovi schopenhauriani" non tanto insistono. sugli elementi che la storia possa fornire e i presagi che sia lecito derivarne, quanto sulle amare delusioni a cui il luogo ed affannoso esercizio. della volontà e dell'istinto vitale dovrà necessariamente ccndurre, e sul finale ravvedimento umano che ne conseguirà. Anche prescindendo da quel processo naturale di fatale estinzione della vita e quindi della. specie umana sulla faccia della terra pèr le forze che operano sul sistema solare, di cui ci parlano i naturalisti, l'umanità sarà condotta ad una. forma di consapevole rinuncia alla vita, dalla coscienza medesima della inanità dei suoi sforzi millenari per conseguire la felicità, da una specie di volontà di liberazione interiore dall'errore fondamentale che vizia le nostre abitudini, i sentimenti nostri, e tutta, anzi, la nostra. visione e valutazione del mòndo: onde l'umanità non avrà pace se non quando sarà contenta di spegnersi e avrà ucciso in sè medesima il desiderio della vita; e prima che il mondo sia effettivamente estinto ella avrà già spento lo spirito che l'ha creato. Ora qual'è questo errore: fondamentale?

II.

Ad una civiltà ancora impaziente di agire e di vivere, come la nostra, ancora spensierata e confidente di conseguire col suo lavoro la felicità, è malagevole il dire la parola austera della saviezza suprema, che può suonare sconforto e aver ,savore di forte agrume". Ma i vecchi dell'oggi saranno i giovani del domani o almeno del lontano 
avvenire : e chi annunzia una riforma dei valori tradizionali del pensiero e della vita, deve prepararsi a dover dire col Marçhese di Posa nel Don Carlos dello Schiller:

\section{Ich lebe}

Ein Bürger derer, welche kommen werden.

Ora non è certo blandire l'età nostra il combattere ogni egoismo, ogni smoderata bramosia di godimento, in cui degenera il sentimento della vita, così vivo nelle presenti generazioni. Ma da questo nobile proposito al volere estirpare 0 annullare ogni desiderio 0 istinto di vita come, immeritavole e indegno, ci corre un ben lungo tratto. La vita, si dice, implica distinzione, individuazione, e perciò antago-' nismo. Ella è attività ad esclusivo beneficio del singolo, dell'individuo, del gruppo, con sacrificio degli altri, o perciò è irrazionale ed immorale. Già nelle stesse forme elementari, la vita si manifesta nel costituirsi che fa una parte della materia centro di attrazione degli elementi eircostanti, con processi di assorbimento e di assimilamento di altra materia; nel formarsi, cioè di un nucleo che tende a mantenersi, ad aecrescersi e riprodursi (il che è tutt'uno) a danno altrui. Nella vita etico-sociale questo processo vitale diviene abuso, sfruttamento, sopraffazione: e di qui lotta di classe, conflitto di nazionalità, avidità economica, monopolii, guerre armate, e via dicendo. Ora l'uomo in quanto è pensiero e ragione, cioè in quanto è essenzialmente uomo, non può non riconoscere che la cagione di tanti danni è alla radice stessa della pianta vitale, nella diversità, nella individuazione, nella competizione che l'alimentano. Questo che Rabindranath-Tagore lamenta oggi stato d'animo e condizione dolorosa della civiltà occidentale, in confronto dell'oriente unificatore, cioè d'essere vita di „quei' che un muro ed una fossa serra“", è, per altri, la condizione comune ad ogni forma di vita. Di qui la necessità razionale di ridurre le dif-' ferenze, eliminare le diversità, far valere l'universale, cioè negare lo stesso fatto vitale. Lo sentirono e lo intravidero anche i mistici dei secoli cristiani. La fede dei nostri padri, difatti, non sarebbe che il pensiero anti-vitale (cupio dissovi, ama nesciri) ancorchè abbellito di qualche immagine vitale, a cui l'uomo non sa mai rinunciare. Dio non è che il Punctum aequans, in cui tutte le diversità apparenti nel mondo si fondono e scompaiono. Così si spiega la vertigine d'annullamento, l'anelito all'identità onde son pervasi i mistici, e il loro amore di tutte le creature in Dio. Perciò anche la santificazione 
del dolore (il cristianesimo è la religione che santifica il dolore); come quello che ci distacca dalla vita, cioè ci aiuta a combattere la degenerazione vitale dell'umanità: E poichè quello che lega l'uomo alla vita è il fascino che ella esercita su di lui per mezzo del piacere, dell'illusione e della bellezza, che invincibilmente lo adescano e lo corrompono, de qui l'avversione che i neo-schopenhauriani mostrano per ogni estetismo, per la sopravalutazione della bellezza e dell'arte. Chi ideò la basilica d'Assisi, e Giotto e gli altri che la decorarono, deviareno dal pensiero dell'umile Francesco, il quale sapeva come le forme e gli adornamenti divertissero l'anima della sua via, che è l'abbandono di sè stessa in Dio, e il distacco da ogni cosa terrena, "l'ebbrezza del dissolvimento" come cantò il Carducci. L'assoluto soltanto soddisfa a questo profondo bisogno che l'uomo ha dell'unità e dell'identità, cioè il bisogno antivitale; e il significato fondamentale della redenzione cristiana sta appunto in questo: nel disciogliere i vincoli che trattengono l'uomo entro limiti egoistici (famiglia, comunità, parentado, tribù) che suddividono l'umanità in tanti elementi o gruppi separati l'uno dall'altro. Il naturalismo pù coll'adorazione della natura aver ravvivato lo smisurato amore alla vita, di cui la recente guerra è il malefico prodotto: ma ,,verrà giorno, sia pur lontano, in cui dall'America, sfinita e convulsa, intossicata dall'orgia vitale, cadente sotto il peso della ropria grandezza, stanca della complicazione dei suoi meccanismi sociali ... da questa terra disillusa e sazia, s'inizierà una grande corrente migratoria ... e il centro di gravità spirituale ci trasporterà nell'Asia primigenia e, come da un suolo rovente verso un paese di frescura e di serenità, gli uomini escirannio a poco a poco dal continente dove la follia umana arrà vanamente grandeggiato come non mai, per andare a mopire placidi e savî, in quello delle grandi ed antiche aspirazioni all'annichilamento; e sarà l'ultima e vera redenzione dell'umanità, la liberazione dalla volontà di vità, errore fondamentale, e funesto, che sola ci può dare, dopo tante esperienze di guerra; la pace anelata".

\section{III.}

Chi ora dovesse ricercare a fondo la consistenza di questo ordine d'idee, e la sua corrispońdonza coi Da ti della psicologia e della ragione crițica, dorrebbe distendere ben più lungo discorso di quello che ci 
sia lecito qui. Non è il caso di ritornare su quella dualità antitetica di vita e di razionalità o intelligenza; sulla quale ebbi altra occasione d'intrattenermi ${ }^{10}$ ). Questa ,divisione dell'uomo in due ",come la chiama il Fouillée, sebbene stia al fondo delle dottrine recenti del Ribot, del James, del Bergson e di altri, contrasta colla più evidente esperienza psicologica; la quale c'insegna che lá vita non è in se irrazionale, e che lo spirito non solamente non esclude da sè la vita, ma ne è anzi la più sublime espressione. Le sottili indagini psicologiche, specialmente del Bergson, sono riuscite a porre in luce la presenza del fattore volitivo ed attivo anche nelle più alte ed astratte operazioni intellettuali. Si tratta qui piuttosto di vedere se l'impulso vitale sia sempre istinto di sopraffazione e di appropriazione, o non piuttosto moto di cooperazione e di consenso. Da un lato, il processo vitale si presenta come uno sterminio continuo degl'individui a beneficio della specie, che è un universale: e perciò è processo costruttivo e continuo; il che vuol dire che è un processo implicitamente razionale o guidato dalla razionalità superiore. Salvochè la razionalità vi è come iscritta e virtuale, dove nel mondo umano questa oscura razionalità della vita diviene e si fa consapevole. Dall'altro lato poi, il frutto più alto della evoluzione vitale è appunto la formazione della personalità umana. Nella sua massima concentrazione, che è la coscienza, la vita raggiunge un segno in cui la massima singolarità si confonde colla massima universalità (la persona); poichè, come per gli stoici antichi, il regno della più alta individualità (il sapiente) è quello pure, per dirla col Gioberti, della più universale Cosmopolitia. Nell'organismo fisieo le parti, o gli elementi vivono della vita e per la vita del tutto, che è rispetto ad essi un universale: nell'organismo sociale la totalità vive anche per la vita dei singoli. Ma come questi non sono soltanto unità bensi persone, cosi in queste è la ragione dell'universalità; dacchè le persone, come diceva il Kant, non possono essere mezzi nè agli altri nè alla totalità collettiva, bensì sóno fini in sè stessi e $a$ sè stessi, e costituiscono un sistema universale di fini. In ogni grado o forma evolutiva della vita, adunque, la competizione e la sopraffazione non è già un obbedire alle leggi della vita, ma è un violarle e negarle; e perciò l'ammonimen o che scaturisce dall'antico apologo di Menenio Agrippa non è diverso da quello che ci viene dalla recente

10) Vedi la ",crisi del pensiero moderno“, in Nuova Antologia, 1914. 
guerra mondiale; la quale non è già il resultato nefasto della volontà normale della vita, bensi di una deformazione di quel sentimento, cioè di un disordine creatosi nel seno della società civile, col prevalervi degli egoismi nazionali e degli istinti di dominio e di oltrepotenza, che sono antivitali e mortiferi. Vita non è, perciò, organamento 0 individuale 0 collettivo, per la cattura o per l'oppressione altrui: ma, nell'ordine tisico, è armonia, coordinamento, accordo di funzioni, nell'ordine morale e sociale è forma di giustizia, sì negli ordini civili e sì nei rapporti internazionali. La distruzione e la morte che accompagna, come sua condizione, l'evoluzione vitale non è mai a beneficio di un individuo o di un nucleo, se non in contingenze anormali. Fella stessa legge della prevalenza 0 sopravvivenza del più forte 0 del più adatto, è inerente un principio di ragione universale, cioè la preservazione della specie e del principio vitale: e dalle stesse guerre onde fu insanguinata la storia umana, anchorche mosse da istinti antivitali, esci pur sempre un nuovo e migliore ordine di cose. Onde se dalla recente ecclissi civile, colla futura Società delle nazioni, dovesse erompere più fulgida la luce della umanità e dạile grandi rovine dovesse sorgere l' „edificio altero e santo“, bene sarebbe stato che questo scandalo fosse arvenuto. E non sarebbe gran danno che il primato e la direzione civile fosse passato, in conseguenza di questa guerra e di questa pace, dall'Europa all'America.

Queste rinnovate dottrine che ricercano nella volontà della vita la radice di ogni male e ne auspicano perciò la totale estirpazione, s'incontrano sostanzialmente con quelle professate p.e. dal Tarde, dal Paulhan, da Max Nordau e da altri ${ }^{11}$ ), pei quali la moralità è fondata sopra convenzioni, o illusioni o menzogne sociali. La letteratura recente, del Thackeray all'Ibsen, dal Tolstoi a Bernardo Shaw ha porto copiosa materia, colle sue veementi critiche antisociali, a codeste dottrine. Nè puo negarsi che e quelle censure e queste teorie non contengono in se molte e dolorose vetità nella loro diagnosi della vita sociale odierna. Ma quando invece si dimostri che la moralità. è la più alta funzione ed espressione normale della vita, cioè della. piena umanità; che ella è fondata non sopra una finzione, ma sopra. leggi pșicologiche necessarie ed inerenti alla natura umana, è salva.

11) V. per tutti il bel libro di P. Gauthier, La Pensée Contemporaine (les Grands Problèmes). Paris 1911, specialmente la p. 175 e segg. 
altresi la - razionalità della vita. Non vi è menzogna senza veiità implicitamente riconosciuta; e la menzogna nulla fonda e nulla ha fondato mai. Cosi se la vita fosse soltanto distruzione degli altri ed egoismo, non avrebbe creato nè l'arte, nè la morale, nè la convivenza civile. Tutti sanno che la bellezza e l'arte non solo è espressione di vita, ma anche funzione essenziale della vita; e percio fa parte di un disegno divino, che da Platone a Dante, dallo Shelley all'Hegel, i più solenni spiriti hanno sempre riconosciuto. Lo stesso poverello d'Assisi, che voleva i suoi compagni servissero al Signore in la etitia, cantava, egli trovatore di Dio, le laudi delle creature, non per annullarle ma per conservarle, e perchè la loró stessa mirabile varietà magnificara, agli occhi suoi, la divina opera creatrice nel mondo.

Se la moralità non è negazione della vita ma perfezione della vita: e se questa è capace di creare, nei suoi più alti fastigi, dei valori eterni di bellezza e di bene, è assurdo il credere che la ragione umana debba esser chiamata ad estinguere questa pura fiamma vitale. Essa è già di per sè inestinguibile ${ }^{12}$ ). Quando attinge cósi alti vertici, ha in sè le ragioni e i titoli della propria industruttibilità; come quella che attiene non più alla terra e alle condizioni contingenti ed efimere che questa offre alla durata della vita fisica, cioè organica, ma ad altre direzioni e dimensioni della realtà. E la realtà universa, nella. cui economia l'individuo-persona, cioè la coscienza (singolare ed universale) ha la sua ragione d'essere, contiene anche le condizioni della perpetuità vitale. Perchè questo attendere soltanto dalla storia e dall'avvenire dell'umanità un'opera quasi di correzione e di revisione ultima delle condizioni e dei fondamenti ,posti da natura“, è una vana chimera. Se vi è questa opera correttiva, cioè la sommissione del talento, o degl'impulsi sensibili e passionali, alla ragione, non è data da un progresso storico da un perfezionamento umano, bensi è un opera immanente e continua in ogni individuo $\mathrm{e}$ in ogni età. Ricordiamoci che il siștema del mondo gravita non sulla povera umanità effimera sulla faccia stessa della terra, ma sulla persona umana, sull'individuo consapevole, che sfugge alle ecatombi umane, come la recente guerra, alle periodiche catastrofi telluriche, e alla stessa opera di volontaria diserzione della vita, perchè ha la sua fina-

$\left.{ }^{12}\right)$ Si veda, fra gli altri, anche l'ultimo romanzo del Wells, The Undying Fire, London, Cassell, 1919. 
lità immortale nell'infinito. Può darsi che la storia abbia un.camm ino ciclico, un periodo segnato sulla faccia della terra, e che un giorno questo sia ưn circuito chiuso. Ma ella sarà stata una gran disciplina, una educazione divina dell'umanità per gl'individui, che troveranno il loro esodo, la loro via di escita e di libertà nella immancabile continuità della vita, in un diverso ordine di esistenza, non opposto al nostro ma contiguo al nostro, sebbene a noi ora inafferrebile, o solo in parte ed imperfettamente comunicabile. Perciò lo spirito non è antivitale ma ultravitale; nel senso che è la più alta forma della vita universa, cioè quella in cui la forza istintiva si ordina e si disciplina, non già si estingue. Tale è il significato, la vera luce, la finalità della coscienza. Nella quale si avvera soltanto la continuità della vita; non già perchè la neghi o comprima, ma perchè la prosegue in sè $\mathrm{e}$ la eleva ad una altezza ove la luce splende perenne ed inestinguibile. Leonardo da Vinci diceva saviamente , chi non stima la vita, non la merita". Se l'umanità giungesse un giorno a non pregiare la vita, non ne sarebbe degna; e non sarebbe mai la luce della ragione che ve l'avrebbe condotta, bensì il suo oscuramento; dacchè l'esercizio stesso dell'intelletto e l'attività dello spirito, come aveva pur detto il vecchio Aristotele, è la massima illuminazione delle vita, come quella che ne illustra le ragioni. Vi possono essere della anime e delle generazioni che giungono a Propter vitam vivendi perdere causas, e conquistando il mondo non guardano a ,perdere l'anima loro"; Ma codesto è non la sostanza e l'affermazione, bensì l'oscuramento e lo smarrimento delle vie della vita; è l'ora tragica della „potenza della tenebre“, che prelude alla luce, e della morte che aspetta e prepara la resurrezione immancabile. 